\title{
EXCLUSIÓN SOCIAL: EXPLORANDO LA DIMENSIÓN EDUCATIVA EN ARGENTINA
}

\author{
María María Ibáñez Martín, ${ }^{2}$ María Marta Formichella ${ }^{a}$ \\ y Lucas Ezequiel Costabel ${ }^{\mathrm{b}}$
}

Fecha de recepción: 4 de marzo de 2019. Fecha de aceptación: 4 de octubre de 2019.

$$
\text { https://doi.org/10.22201/iiec.20078951e.2020.200.68518 }
$$

Resumen. La exclusión social es un proceso complejo y multidimensional que indica la persistencia de privaciones en dimensiones relevantes de la vida, entre ellas, la educación. Bajo este contexto, el presente trabajo estudia los determinantes de la exclusión en la dimensión educativa, específicamente en su grado original (acceso) y busca evaluar si varían en relación con las condiciones socioeconómicas de la población objetivo. Para ello, se estiman modelos de regresión logística utilizando datos de la Encuesta Permanente de Hogares (EPH) de Argentina, así como información obtenida de un relevamiento propio llevado a cabo en abril de 2016 en tres barrios vulnerables de Bahía Blanca (Argentina).

Palabras clave: Argentina; exclusión social; educación; modelos logísticos; encuesta de hogares.

Clasificación JEL: C25; C35; I21; I24; I30.

\section{SOCIAL EXCLUSION: EXPLORING THE EDUCATIONAL DIMENSION IN ARgENTINA}

\begin{abstract}
Social exclusion is a complex, multidimensional process which points to the persistence of a variety of deprivations in key dimensions of life, education among them. Working within this context, this paper studies determinants of exclusion in the educational dimension, specifically from its original degree (access), and seeks to evaluate whether they vary in relation to the socioeconomic conditions of the target population. To do this, logistic regression models were calculated using data from Argentina's Permanent Household Survey (PHS), in addition to information obtained from our own survey, performed in April of 2016 in three vulnerable communities of Bahía Blanca, Argentina.
\end{abstract}

Key Words: Argentina; social exclusion; education; logistic models; household surveys.

\footnotetext{
${ }^{a}$ Instituto de Investigaciones Económicas y Sociales del Sur (IIES, CONICET-UnS), Argentina y ${ }^{b}$ Universidad Nacional del Sur, Argentina. Correos electrónicos: maria.ibanez@uns.edu.ar; mformichella@iiess-conicet.gob.ar y lucas.costabel@hotmail.com, respectivamente.
} 


\section{INTRODUCCIÓN}

La exclusión social se define como un proceso complejo y multidimensional que indica la existencia de privaciones en diversas dimensiones relevantes de la vida (Atkinson y Hills, 1998; Burchardt, 1998; Comisión de las Comunidades Europeas, 1992). Asimismo, implica la incapacidad de los individuos excluidos de participar en las actividades y en los vínculos que, por lo general, están disponibles para los miembros de una sociedad de referencia (Ibáñez Martín et al., 2017).

Entre las esferas que abarcan el concepto de exclusión destaca la educativa, ya que la educación es esencial para que las personas puedan desarrollarse (Sen, 1999). Sin embargo, se evidencian diferentes niveles de esta exclusión. El inicial es la inaccesibilidad, después se contempla la segregación, la inexistencia de condiciones necesarias para participar con éxito en el sistema, el abandono y la inequidad en los resultados, entre otras cuestiones (Formichella, 2010; Krüger, 2013).

En Argentina, en 2006, el Congreso de la Nación sancionó la Ley $\mathrm{N}^{\circ}$ 26.206, denominada "Ley de Educación". Su artículo $3^{\circ}$ explicita: "La educación es una prioridad nacional y se constituye en política de Estado para construir una sociedad justa, reafirmar la soberanía e identidad nacional, profundizar el ejercicio de la ciudadanía democrática, respetar los derechos humanos y libertades fundamentales y fortalecer el desarrollo económicosocial de la Nación”. Establece entonces que:

a) La estructura del Sistema Educativo Nacional comprende cuatro niveles (Educación Inicial, Educación Primaria, Educación Secundaria y Educación Superior) (Ley 26.206, art. 17);

b) La obligatoriedad escolar abarca desde la sala de cinco de nivel inicial hasta la finalización del nivel secundario (Ley 26.206, art. 16);

c) "El Estado Nacional, las Provincias y la Ciudad Autónoma de Buenos Aires tienen la obligación de universalizar los servicios educativos para los/as niños/as de cuatro (4) años de edad" (Ley 26.206, art. 19); y

d) Cada jurisdicción optará por dos tipos de estructura educativa: seis años de nivel primario y seis de nivel secundario, o siete y cinco, respectivamente (Ley 26.206, art. 134).

El acceso a la educación en Argentina cuenta con importantes mejoras desde la década de 1970 hasta los años 2000; siendo las antes mencionadas las más relevantes durante las últimas dos décadas del periodo mencionado 
(Miranda et al., 2007; Krüger, 2013). Si bien en el nivel primario se alcanzó la universalidad en la escolarización, no sucede lo mismo con el nivel secundario, pues más allá de que la desigualdad en el acceso en este nivel es baja (Serio, 2017), es un problema que persiste, en particular en los últimos años del tramo educativo.

A partir de la publicación de la Ley de Educación 26.206 (2006), la asistencia al nivel educativo medio es obligatoria; sin embargo, según datos del último Censo Nacional de Población, Hogares y Viviendas (INDEC, 2010), 34\% de los individuos en edad de asistir a dicho nivel (jóvenes entre 12 y 18 ańos), no lo hacen. Esto representa en términos absolutos más de un millón y medio de personas que se encuentran fuera del sistema. Por ello, se toma como población de análisis a los individuos en edad de asistir al nivel secundario.

El presente trabajo busca responder a la siguiente interrogante: ¿el comportamiento de los factores determinantes de la exclusión educativa en la población vulnerable, es similar al observado en la población general (incluyendo todos los estratos sociales)? Para evaluar cuáles son los determinantes, así como la homogeneidad de su significatividad estadística, se analizan tres poblaciones: Argentina urbana, el aglomerado Bahía Blanca-Cerri (Provincia de Buenos Aires, Argentina) y tres barrios vulnerables de dicho aglomerado. En los primeros dos casos se emplean datos obtenidos de la Encuesta Permanente de Hogares (EPH) del Instituto Nacional de Estadística y Censos (INDEC, 2016), Argentina, mientras que en el caso restante se toma información proveniente de un relevamiento propio llevado a cabo en el marco del Proyecto de Desarrollo Tecnológico y Social (PDTs): "Diseño de estrategias para mejorar las oportunidades educativas de la población vulnerable de Bahía Blanca a través de la ONG Red de Voluntarios" (en el cual participaron las autoras). La selección del periodo temporal se fundamenta en que el trabajo de relevamiento de los barrios se realizó en una única oportunidad (abril de 2016) y los otros dos casos se adecuaron al mismo trimestre del mismo año para poder comparar los resultados (evitando que cambios coyunturales de la economía e institucionales pudieran incidir en el estudio).

Se utiliza como metodología un modelo de regresión logística para Argentina y para los barrios relevados. Mientras que en el caso del aglomerado Bahía Blanca-Cerri, debido a cuestiones técnicas que se explican en la sección específica, se hace uso de un análisis de correlación. El trabajo se estructuró de la siguiente manera: en la sección 2 se presenta el marco teórico; en la 3 se explica la metodología, las variables y los datos; en la 4 se exponen los resultados $\mathrm{y}$, por último, las consideraciones finales. 


\section{MARCO TEÓRICO}

\section{Exclusión social: la exclusión educativa en su primer nivel}

Diversos autores utilizan el término exclusión para referirse a situaciones de desigualdad social o pobreza. Sin embargo, pobreza, desigualdad y exclusión social son fenómenos distintos, con causas y consecuencias diferentes para la sociedad (Ibáńez Martín, 2018).

El proceso excluyente es considerado por algunos investigadores como una condición dicotómica de la sociedad, existiendo individuos incluidos y excluidos (Tezanos, 1999; Lipton et al., 1998), mientras que otros autores consideran que existen situaciones intermedias con privaciones en diferentes esferas y con distinta intensidad (Atkinson y Hills, 1998; Golovanevsky, 2003; Kristensen, 1995; BID, 2007).

La exclusión se puede decir que es un fenómeno multidimensional. Las esferas que intervienen son diversas y depende de lo que es "relevante" para que un individuo desarrolle su vida en una sociedad y tiempo determinado (Comisión de las Comunidades Europeas, 1992; Atkinson y Hills, 1998; Burchardt, 1998). No obstante, existe disenso respecto a la importancia de cada esfera en la conformación de procesos excluyentes. Así, algunos autores consideran que todas las dimensiones cumplen un rol protagónico (Burchardt et al., 1999); mientras que otros destacan una única dimensión: la económica (Minujin, 1999; Castells, 2001); la laboral (Comisión de las Comunidades Europeas, 1992, 2000; Kessler, 2014); la política (Silver, 1994; Berghman, 1995; Marshall, 1964); o bien la participación ciudadana (Fabre, 2000; Atkinson y Hills, 1998).

El trabajo opta por definir el fenómeno de exclusión con base en lo que Kessler (2011) denomina una concepción pluralista. Esto implica la multidimensionalidad y, a su vez, la existencia de matices de exclusión. Estivill (2003) expresa que la detección de éstos es relevante porque las medidas de acción que deben realizarse sobre los grupos son distintas, de lo contrario podrían existir efectos perversos.

Siguiendo a Ibáñez Martín (2018), se considera que la educación es una de las dimensiones centrales en la explicación de los procesos de exclusión social. Algunos autores destacan que el grado de educación es determinante del desempeño del resto de las dimensiones (Rama, 1983; Vitello y Mithaug, 1998; Rivero, 1999; Látas, 2002; Brugué et al., 2002; Blanco, 2006; Sarrionandia, 2006; Hopenhayn, 2008; De la Puente, 2009; Kessler, 2011; Sánchez, 2012; 
Ibáñez Martín, 2015) y la Organización de las Naciones Unidas para la Educación, la Ciencia y la Cultura (UNESCo) define a la inclusión educativa como el proceso que permite identificar y responder a la diversidad de las necesidades de todos los estudiantes. Así, la inclusión educativa involucra cambios con una visión común que incluye a los individuos del rango de edad apropiado y la convicción de que es la responsabilidad del sistema educar a todos (UNESCO, 2006). Según Narodowski (2008), incluir significa reunir los esfuerzos de distintos sectores de la sociedad para brindar una educación sensible a las necesidades específicas de cada uno, compensando las desigualdades y facilitando el acceso, la permanencia y el progreso a quienes más lo necesiten.

Así, la inclusión educativa no es una cuestión dicotómica: mientras que el primer grado de inclusión se refiere al acceso y puede definirse de ese modo, el resto representa cuestiones de calidad, retraso y logros, entre otros aspectos, que se definen a partir de grados (Terigi et al., 2010; Morresi et al., 2017).

\section{La exclusión educativa en su grado original: antecedentes en Argentina}

Diversas investigaciones han estudiado los determinantes de la asistencia escolar de nivel medio en Argentina. Sosa y Marchionni (1999) analizan el tema utilizando datos de la EPH del ańo 2006 de los aglomerados Gran Buenos Aires, La Plata y Mendoza en 1997 y destacan que el ingreso, la educación de los padres (en especial de la madre) y la estructura familiar afectan de manera importante la asistencia.

Por su parte, Bertranou (2001) a partir de la Encuesta de Desarrollo Social investiga y encuentra que la probabilidad de asistencia aumenta con el ingreso, la educación de los padres (más con la de la madre) y el género femenino. Resultado encontrado también por Miranda (2010), quien analiza la relación entre el género y la asistencia a la escuela secundaria con base en datos del Censo Nacional de Población y Vivienda 2001 y de la EPH del año 2006. Asimismo, Bertranou (2001) destaca que la probabilidad de asistencia se reduce con la cantidad de hermanos, el hecho de que la cabeza de hogar sea mujer y la incorporación de los jóvenes al mercado laboral; mientras que Sosa y Marchionni (1999) y De Vos (2001) encuentran que dicha probabilidad es mayor para quienes habitan hogares con ambos padres y casados.

Gasparini (2001) analiza la injusticia generada en la desigualdad de resultados vinculada a factores inaceptables, como el género y el ingreso. Para ello, a partir de datos de la EPH del año 2006, se concentra en el caso de la asistencia escolar de nivel medio en Gran Buenos Aires y otras ciudades de Argentina. 
Cerrutti y Binstok (2004) estudian el abandono escolar de jóvenes entre 15 a 19 años con base en datos de la Encuesta de Desarrollo Social de 1997, y concluyen que los principales determinantes son: el clima educativo del hogar (efecto negativo), la estructura familiar (efecto negativo de estar ambos padres presentes), la cantidad de hermanos (efecto positivo), la presencia de nińos pequeños (efecto positivo) y existencia de Necesidades Básicas Insatisfechas (NBI) (efecto positivo).

Mientras que Groisman y Calero (2010) y Groisman (2011) analizan los determinantes de la escolarización y la participación laboral en Argentina para jóvenes entre 15 y 18 años. Los autores encuentran que la probabilidad de asistencia a nivel medio es menor para los varones, para quienes no residen con sus padres, así como para quienes están más cerca de la adultez y para quienes viven en hogares pobres, monoparentales o con mayor cantidad de miembros (más si son niños pequeños). También destacan que la probabilidad de permanecer en la escuela es mayor para jóvenes que habitan hogares con un miembro ocupado formal. Paz y Cid (2012) también encuentran que la asistencia se relaciona positivamente con ser mujer y el clima educativo del hogar; y negativamente con la edad, con el hecho de que el jefe del hogar se encuentre desempleado o tenga trabajo informal, con la cantidad de miembros y con el número promedio de privaciones.

Recientemente, Formichella y Krüger (2019) y Alderete et al. (2017) investigan acerca del acceso a la escolarización y las condiciones de educabilidad de adolescentes habitantes de barrios vulnerables de la ciudad de Bahía Blanca. Las primeras autoras proponen un estudio de clústeres y encuentran que los hogares con presencia de ambos padres o con jefa de hogar son favorables para la educación de sus adolescentes. También, hallan que el tipo de actividades que se realizan en las familia son relevantes: el no abandono se vincula con mayor frecuencia a actividades deportivas, de lectura o culturales; mientras que se asocia negativamente con mayor frecuencia a el uso de Internet, el jugar a videojuegos o mirar televisión. Las segundas investigadoras encuentran que ser mujer, usar Internet y poseer un mayor clima educativo en el hogar aumentan las posibilidades de acceso; mientras que habitar un hogar con NBI o ser mayor en edad las disminuyen. Los resultados encontrados para la población de barrios vulnerables no difieren de los reportados en otras investigaciones que han analizado la cuestión de la asistencia escolar en la población urbana total (vulnerable y no vulnerable).

Con la presente investigación se pretende contrastar lo hallado en los últimos trabajos antes mencionados, por ejemplo, que las variables que afectan la asistencia escolar en contextos de privaciones multidimensionales coinci- 
den con las que lo hacen en contextos globales. Sin embargo, incorpora dos novedades, por un lado, se plantea un modelo idéntico para la muestra de la población total urbana nacional y para la correspondiente a los barrios vulnerables analizados, lo cual hace que las conclusiones sean más robustas; por otra parte, se incorpora el análisis a nivel de aglomerado urbano, considerando en específico en el que están insertos los barrios en cuestión. Esto último permite estudiar los determinantes de la asistencia en dos poblaciones que comparten las condiciones generales vinculadas a su zona geográfica, evitando los sesgos que pudieran existir al trabajar con la población a nivel nacional sin controlar por las características locales.

\section{METODOLOGÍA, VARIABLES Y DATOS}

\section{Metodología de estimación: modelo de regresión logística}

Las situaciones o problemas reales caracterizados a través de variables categóricas, incumpliendo con la condición de continuidad, pueden analizarse y estimarse a partir de modelos de regresión logística (Williams, 2006). El problema abordado en el presente trabajo cumple con dicho requerimiento, puesto que el foco de análisis es el primer grado de exclusión educativa (acceso).

El objetivo principal de los modelos logísticos es estimar la probabilidad de que un evento suceda a partir de determinadas variables explicativas (Liao, 1994). Permiten conocer el sentido, en caso de existir, de la relación entre éstas y el suceso a explicar (Velasco, 1996).

Los modelos logísticos suponen que el logaritmo de la razón de probabilidades está linealmente relacionado con las variables regresoras, principal diferencia con el modelo de probabilidad lineal ${ }^{1}$ (Gujarati y Porter, 2009). El modelo puede expresarse a partir de la siguiente forma funcional:

$$
\log i t\left(p_{i}\right)=\ln \left(\frac{p_{i}}{1-p_{i}}\right)=\beta_{0}+\beta_{1} X_{1, i}+\beta_{2} X_{2, i}+\ldots+\beta_{k} X_{k, i}
$$

Donde los parámetros que acompañan a las variables explicativas son estimados a través de máxima verosimilitud y $p_{i}$ es la probabilidad de que el evento bajo interés suceda.

1 El modelo de probabilidad lineal tiene numerosas críticas para los modelos con variable dependiente dicotómica, que escapan al objetivo de la investigación. Para mayor detalle véanse Gujarati y Porter (2009). 
Aquí, resulta adecuado aplicar modelos logit para estimar el efecto de las características familiares e individuales sobre la exclusión en el sistema educativo. Así, la variable dependiente que se denomina Asistencia es dicotómica, toma valor 1 si el alumno asiste a un centro educativo y 0 en caso contrario.

La interpretación de los modelos de regresión logística es difícil debido a que los estimadores indican la variación de la razón de probabilidades ante un cambio en las variables explicativas (Gujarati y Porter, 2009). Como señalan Pindyck y Rubinfeld (1998), el efecto de cada variable individual depende de su valor inicial y del valor que toman las demás variables explicativas incorporadas al modelo.

En función del objetivo de este trabajo, los regresores de mayor interés son los vinculados al nivel socioeconómico del hogar como: nivel de ingresos, estatus ocupacional y clima educativo. Sin embargo, dado que pueden existir otros factores que afecten al mismo momento a la variable dependiente no sería correcto, desde el punto de vista estadístico, omitir otras variables que la evidencia previa y la teoría muestran como determinantes. Si dicha omisión se realizara el efecto de estas variables podría quedar incluido (erróneamente) dentro de aquel que se vincula a las variables centrales del estudio. De esta manera, son incorporadas sobre todo para controlar sesgos de tipo estadístico (Wooldridge, 2010).

Los modelos logísticos son estimados para la muestra restringida ${ }^{2}$ de la EPH para todos los aglomerados de Argentina y para la muestra correspondiente a los tres barrios vulnerables de la ciudad de Bahía Blanca que fueron estudiados el marco del mencionado PDTs. En el caso de la submuestra de la EPH correspondiente al aglomerado Bahía Blanca-Cerri, no es factible estimar modelos de tipo logístico, debido al acotado número de observaciones que se obtienen al restringir la muestra por grupo etario y la escasa variabilidad de las variables en ese subconjunto.

Como estrategia alternativa se estima un modelo Tobit, debido a que la distribución de la variable dependiente se encuentra altamente concentrada en la cola izquierda de la distribución y se reconoce su bondad de ajuste ante estas situaciones y muestras pequeñas (Jiménez Zambrano, 2005). Sin embargo, el modelo no converge por falta de observaciones. Entonces, con fines descriptivos, se aplican test de correlación entre la variable dependiente y las explicativas trabajadas en los modelos logísticos estimados para las otras dos poblaciones, con fin de poder comparar las tres poblaciones objetivo del trabajo.

2 Dado que la población objetivo son aquellos que asisten al nivel secundario, la muestra se restringe a la población que se encuentra en el grupo etario comprendido entre 12 y 18 años. 
De nuevo, la cantidad de observaciones delimita el test posible de aplicar. En este caso, la correlación es analizada a partir del coeficiente tau-b de Kendalls's, siendo una segunda opción a la correlación de Spearman. Dicho coeficiente se comporta mejor que $C h i^{2}$ en muestras pequeñas.

\section{Variables}

Para los análisis propuestos se utilizan variables relativas a características individuales de los alumnos y de los hogares que provienen. A continuación, se describen. En el cuadro 1 se presentan las estadísticas descriptivas.

\section{Variables individuales}

- Sexo: variable dicotómica que toma valor 1 si el estudiante es varón y 0 en caso de ser mujer.

- Edad: variable continua que indica los años cumplidos del alumno.

- Adolescente activo: variable dicotómica que toma valor 1 si el adolescente se encuentra ocupado o desocupado y 0 en caso contrario.

- Cobertura salud: variable que toma valor 1 si el individuo cuenta con cobertura en salud de algún tipo y 0 en caso contrario.

- Tarea doméstica: variable dicotómica que toma valor 1 si el adolescente declara ser el principal responsable de realizar las tareas de la casa y 0 en caso contrario. Se consideraron aquellos casos donde el reporte sobre la cantidad de horas que el individuo dedica a dichas tareas supera las dos horas diarias.

- Adolescente cónyuge: toma valor 1 si el adolescente es identificado como el componente cónyuge del hogar y 0 en caso contrario.

\section{Variables familiares}

- Hogar monoparental: es una variable dicotómica que toma valor 1 si el jefe de familia no posee cónyuge y 0 en caso contrario.

- Clima educativo del hogar: variable continua que representa el promedio de años de educación alcanzado por los mayores de 18 años del hogar (SITEAL, 2015).

- Estatus ocupacional jefe del hogar: informa sobre la situación laboral del jefe del hogar según las siguientes categorías: trabajador formal; trabajador 
informal; trabajador desocupado y trabajador inactivo. Siendo esta última, la categoría base en las estimaciones de los modelos logísticos. El INDEC define como desocupado a todo individuo mayor de 10 años que no trabajó en la semana de referencia, pero estuvo disponible y buscó activamente trabajo, y como inactivo a quien no trabajó ni buscó trabajo. Los trabajadores informales comprenden a los trabajadores asalariados no registrados y a los cuentapropista y patrones cuyos ingresos per cápita familiar pertenecen a los cinco primeros deciles de la distribución (Formichella, 2011).

En la muestra correspondiente al PDTs, en función de la información disponible, se utiliza la variable "hogar con jefe trabajador inestable", la cual es dicotómica, toma valor 1 si el jefe de hogar tiene un trabajo sin seguridades en relación con la continuidad en el tiempo y 0 si tiene un trabajo formal, está desempleado o es inactivo.

- Hacinamiento: es una variable dicotómica que toma valor 1 si en el hogar hay más de tres personas por cuarto (INDEC, 2014) y 0 en el caso contrario.

Cuadro 1. Estadísticas descriptivas de variables explicativas de la probabilidad de asistencia a centro educativo. Segundo trimestre 2016

\begin{tabular}{|c|c|c|c|}
\hline & $\begin{array}{c}\text { Población urbana } \\
\text { Argentina (EPH) }\end{array}$ & $\begin{array}{l}\text { Bahía Blanca-Cerri } \\
\text { (EPH) }\end{array}$ & Barrios PDTS \\
\hline & $n=6997$ & $n=127$ & $n=201$ \\
\hline \multicolumn{4}{|l|}{ Variables familiares } \\
\hline Hogar monoparental & $30.62 \%$ & $32.28 \%$ & $20.90 \%$ \\
\hline Clima educativo del hogar & 10.82 & 10.77 & 8.57 \\
\hline Hacinamiento & $7.79 \%$ & $3.94 \%$ & $19.90 \%$ \\
\hline \multicolumn{4}{|l|}{ Estatus ocupacional } \\
\hline Formal & $49.18 \%$ & $48.82 \%$ & - \\
\hline Informal & $34.76 \%$ & $51.18 \%$ & - \\
\hline Desocupado & $3.47 \%$ & $4.72 \%$ & - \\
\hline Inactivo & $19.12 \%$ & $11.02 \%$ & - \\
\hline Inestable & - & - & $38.31 \%$ \\
\hline
\end{tabular}




\begin{tabular}{lccc}
\hline & $\begin{array}{c}\text { Población urbana } \\
\text { Argentina (EPH) }\end{array}$ & $\begin{array}{c}\text { Bahía Blanca-Cerri } \\
\text { (EPH) }\end{array}$ & Barrios PDTS \\
\cline { 2 - 4 } & $n=6997$ & $n=127$ & $n=201$ \\
\hline Variables individuales & $50.78 \%$ & $51.97 \%$ & $57.51 \%$ \\
Sexo & 15.07 & 14.73 & 14.62 \\
Edad & $10.13 \%$ & $13.39 \%$ & $2.00 \%$ \\
Adolescente cónyuge & $5.66 \%$ & $4.72 \%$ & $9.09 \%$ \\
Adolescente activo & $2.02 \%$ & $3.94 \%$ & $65.45 \%$ \\
Tarea doméstica & $63.81 \%$ & $58.27 \%$ & $34.50 \%$ \\
Cobertura salud & & & \\
\hline
\end{tabular}

Fuente: elaboración propia con base en EPH (INDEC, 2016) y relevamiento del proyecto PDTS. Stata 13.1.

\section{Datos}

Los datos utilizados provienen de dos fuentes de información. Para el caso de Argentina y del aglomerado Bahía Blanca-Cerri se toma la EPH, mientras que para el caso de la población vulnerable se toma información proveniente del relevamiento propio.

La EPH es un programa nacional de Argentina dedicado a producir de manera sistemática y continua indicadores socioeconómicos y demográficos. Es llevado a cabo por el INDEC, actualmente cubre 31 aglomerados urbanos y sólo uno urbano-rural, así su representatividad se vincula exclusivamente al sector urbano (INDEC, 2003). Si bien sería interesante poder incluir el sector rural, en Argentina no hay bases de microdatos anuales disponibles que permitan realizar el análisis.

En la construcción de la muestra de los hogares a encuestar por la EPH se aplican técnicas estadísticas muy rigurosas que hacen posible que se obtengan datos con precisión. La muestra de los hogares a encuestar por la EPH incluye 25 mil hogares por trimestre, lo cual implica 100 mil hogares al ańo, ya que la muestra tiene representatividad a nivel de cada aglomerado y también a nivel nacional (INDEC, 2003).

El proyecto en el cual se enmarca el relevamiento propio tiene por objetivo elaborar un plan estratégico para la ONG "Red de Voluntarios por una Infancia Mejor", la cual desarrolla tareas de intervención con el fin de promocionar los derechos de la infancia y de la adolescencia. Así, el PDTs se orienta a realizar 
un aporte con el fin de mejorar las intervenciones de la ONG vinculadas a la educación en tres de los barrios periféricos en los que trabaja.

El relevamiento consistió en la realización de encuestas a los hogares de los barrios "Stella Maris", "Nueve de Noviembre" y "Cabré More". Dado que estos barrios pertenecen a la localidad de Bahía Blanca, se ponderó interesante comparar su situación con la de la ciudad que los alberga y con el total del país. Para que la comparación no se viera afectada por sesgos provenientes del periodo de referencia considerado o de la época del año calendario analizada, se tomaron datos del segundo trimestre de 2016 (el cual incluye el mes de abril de 2016, tiempo en que se llevó a cabo el relevamiento en el marco del PDTs).

La encuesta a hogares realizada por medio del PDTS se basó en dos formularios: uno referido al hogar y la vivienda, y otro individual, aplicado a todos los miembros de entre 3 y 18 ańos enfocado en conocer cuestiones vinculadas a su educación. La muestra se construyó en un proceso de dos etapas y usando un probabilístico de manzanas y hogares; a partir de la misma se relevaron 381 hogares que contaban con 1459 personas. De tal manera que la muestra resultó representativa de la totalidad de la población de los tres barrios periféricos.

\section{RESUlTADOS}

A continuación se presentan los resultados obtenidos de acuerdo con las metodologías propuestas.

\section{Resultados para Argentina}

A partir de la muestra utilizada, la EPH del segundo trimestre de 2016, se obtuvo que $93 \%$ de la población objetivo (individuos entre 12 y 18 años) participa del sistema educativo formal. En el cuadro 2 pueden observarse los resultados provenientes de la regresión econométrica utilizada.

Ciertas variables explicativas de la probabilidad de asistencia son estadísticamente significativas y otras no. Entre las primeras, la mayoría posee una incidencia negativa en las probabilidades de asistir, coincidiendo con lo esperado de acuerdo con la teoría y la evidencia previa. 
Cuadro 2. Determinantes de la probabilidad de asistir al nivel medio de educación. Argentina-Población Urbana. Segundo trimestre 2016

\begin{tabular}{|c|c|}
\hline Variables explicativas & Coeficientes \\
\hline \multirow[t]{2}{*}{ Edad } & $-0.4297^{\star}$ \\
\hline & $(0.0556)$ \\
\hline \multirow[t]{2}{*}{ Sexo } & $-0.3250^{\star}$ \\
\hline & $(0.1587)$ \\
\hline \multirow[t]{2}{*}{ Clima educativo } & $0.2034^{*}$ \\
\hline & $(0.3980)$ \\
\hline \multirow[t]{2}{*}{ Hogar monoparental } & $-0.4717^{\star}$ \\
\hline & $(0.2001)$ \\
\hline \multirow[t]{2}{*}{ Adolescente activo } & $-1.4613^{*}$ \\
\hline & (0.1878) \\
\hline \multirow[t]{2}{*}{ Hacinamiento } & $-0.8770^{\star}$ \\
\hline & (0.1914) \\
\hline \multirow[t]{2}{*}{ Cobertura en salud } & $0.5710^{*}$ \\
\hline & $(0.2440)$ \\
\hline \multirow[t]{2}{*}{ Tarea doméstica } & $-0.6864^{\star \star}$ \\
\hline & $(0.3518)$ \\
\hline \multirow[t]{2}{*}{ Hogar jefe informal } & 0.1039 \\
\hline & $(0.2085)$ \\
\hline \multirow[t]{2}{*}{ Hogar jefe formal } & $0.3842^{\star \star \star}$ \\
\hline & $(0.2173)$ \\
\hline \multirow[t]{2}{*}{ Hogar jefe desocupado } & 0.3857 \\
\hline & $(0.3572)$ \\
\hline \multirow[t]{2}{*}{ Adolescente cónyuge } & 0.3606 \\
\hline & $(0.2965)$ \\
\hline \multirow[t]{2}{*}{ Constante } & $7.3997^{\star}$ \\
\hline & $(0.8140)$ \\
\hline
\end{tabular}

Notas: ${ }^{*} ;{ }^{* *} ;{ }^{* \star \star}$ : variables estadísticamente significativas al 1,5 y $10 \%$, respectivamente. Desvios estándar entre paréntesis.

Fuente: elaboración propia con base en EPH (INDEC, 2016), segundo trimestre 2016. 
Se observa que a mayor edad menor es la probabilidad de asistencia. Esto es de esperarse debido al mayor costo de oportunidad que representa la asistencia escolar -en desmedro de la incorporación al mercado laboral- de adolescentes próximos a cumplir 18 años, en relación con adolescentes menores. Si bien la presión por colaborar con la economía del hogar puede existir en diferentes edades, a medida que los individuos crecen aumentan sus posibilidades de generar ingresos y, de este modo, el costo de oportunidad de destinar tiempo a estudiar es mayor. Aunque en algunos casos podrá evidenciarse la compatibilización de ambas actividades (estudio y trabajo), lo que muestra el efecto de esta variable es que en su mayoría no será así, provocando un efecto negativo sobre la variable dependiente; en clara coincidencia con lo hallado por Paz y Cid (2012) y por Alderete et al. (2017).

De igual forma, las mujeres poseen mayores probabilidades de asistencia que los hombres. Los varones suelen insertarse con mayor facilidad y mejores salarios en el mercado laboral, lo cual aumenta el costo de oportunidad de estudiar. Esto concuerda con lo encontrado por Paz y Cid (2012), quienes afirman que entre 2003 y 2009 los varones asistieron menos a la escuela que las mujeres debido a su condición laboral. Asimismo, Bertranou (2001), Miranda (2010), Groisman y Calero (2010), Groisman (2011) y Alderete et al. (2017) obtienen idéntico resultado en relación con el género.

Respecto a la estructura familiar, al igual que Sosa y Marchioni (1999), De Vos (2001) y Formichella et al. (2017), encuentran que pertenecer a hogares monoparentales tiene una incidencia negativa. Esto también puede fundarse en un mayor costo de oportunidad de asistir a la escuela: por un lado, Cerrutti y Binstock (2004) sostienen que la ausencia de un adulto potencialmente perceptor de ingresos puede presionar al adolescente a tener que generar ingresos; por otro, puede ser que la ausencia de dicho adulto limite las posibilidades económicas para enfrentar los gastos asociados a la educación del joven o bien pueden evidenciarse ambas situaciones. También podría pensarse que la presencia de una irrupción en la familia puede afectar la atención puesta en la educación, aunque la profundización al respecto queda fuera de la esfera del presente trabajo.

La condición de actividad del adolescente también afecta de forma negativa a la asistencia. Como ya se mencionó, el trabajo y la educación compiten por el tiempo de los individuos, convirtiéndose en opciones excluyentes en los casos más severos. Groisman (2012) manifiesta que la inserción en el mercado laboral es un determinante del abandono y retraso escolar.

El mismo efecto puede encontrarse en aquellos adolescentes que son responsables de realizar las tareas domésticas, como del cuidado del hogar. 
Quienes tienen a cargo la reproducción social (todas aquellas actividades de cuidado que garantizan la existencia humana) también tienen mayores probabilidades de no asistir al colegio. Esto es consistente, dado que la reproducción social requiere que los individuos destinen un tiempo equivalente e incluso mayor al requerido en el trabajo que se realiza fuera de los hogares. Esto coincide con lo hallado por Miranda (2010), quien señala que en Argentina es frecuente que las personas que realizan tareas domésticas de forma habitual posean menor presencia en la educación secundaria. Asimismo, es más frecuente que suceda en las familias de bajos niveles de ingresos y en el caso de las mujeres.

Otro de los determinantes negativos de la asistencia escolar es habitar en un hogar con hacinamiento. Herrero (2005) explica que el hacinamiento refleja condiciones habitacionales deficientes, afectando la salud y las posibilidades de que un individuo pueda estudiar; consistente con lo también hallado por Groisman y Calero (2010), Groisman (2011), Paz y Cid (2012) y Alderete et al. (2017).

De igual forma, contar con cobertura de salud y poseer un jefe inmerso en el mercado laboral formal afectan positivamente la asistencia. Si bien ambas variables se relacionan, no son estrictamente coincidentes. En relación con la condición laboral, el efecto positivo puede explicarse por la estabilidad en la recepción de ingresos que permite planificar la educación de los menores de la familia y limita la necesidad de que los adolescentes pasen a formar parte de la PEA. A esta misma conclusión arriban Groisman y Calero (2010), Groisman (2011) y Paz y Cid (2012).

Con respecto al clima educativo del hogar, se observa que cuanto mayor sea, mayor es la probabilidad de asistencia escolar. La relevancia que se da a la inversión en educación tiene una relación positiva con el nivel educativo de los padres (Ibánez Martín, 2015) y por ello es probable que los padres más educados hagan mayores esfuerzos para que sus hijos asistan al colegio. Asimismo, es razonable pensar que adultos más educados poseen más y mejores herramientas para acompañar el proceso educativo de los menores. Este efecto positivo coincide con Sosa y Marchionni (1999), Bertranou (2001), Cerrutti y Binstok (2004), Paz y Cid (2012) y Alderete et al. (2017).

En suma, a nivel nacional la probabilidad de asistencia al colegio de los individuos de entre 12 y 18 años se vincula positivamente con el clima educativo del hogar, con la posesión de cobertura en salud y con la inserción del jefe de hogar en el mercado laboral formal. Por el contrario, avanzar en edad, ser hombre, vivir en un hogar monoparental o con hacinamiento, que el adolescente esté activo en el mercado laboral o bien sea el responsable de 
las tareas domésticas, afecta de forma negativa la mencionada probabilidad. Estos resultados coinciden con investigaciones previas para Argentina.

\section{Resultados para Bahía Blanca-Cerri}

Como fue mencionado en la sección metodológica, debido a la cantidad de observaciones y a la variabilidad de la muestra, para el aglomerado Bahía Blanca-Cerri no fue posible estimar modelos de regresión logística y se realizó un análisis de correlación de variables a partir del coeficiente tau-b de Kendall's (véase cuadro 3). Este permite extraer una conclusión preliminar sobre los factores que explicarían las probabilidades de asistencia.

En este caso el porcentaje de adolescentes que asisten a nivel secundario es de $92 \%$. Los resultados muestran que la significatividad de las variables a nivel aglomerado es mayormente consistente con lo observado para Argentina. Avanzar en edad, ser hombre, estar activo laboralmente y pertenecer a un hogar monoparental tiene incidencia negativa en las probabilidades de que los adolescentes de Bahía Blanca asistan a la escuela. A su vez, si el adolescente posee cobertura en salud aumentan dichas posibilidades. Sin embargo, a diferencia del nivel nacional urbano, se halló que ser cónyuge es una variable estadísticamente significativa que también aumenta la probabilidad de asistencia.

Respecto a la condición de actividad; si el jefe de hogar es desocupado, informal o formal las probabilidades de asistencia disminuyen. Cabe destacar que en este caso la categoría de base es hogar con jefe inactivo (de otra forma el signo de la condición formal pareciera ser contra intuitivo). Si se analizan los coeficientes dejando como categoría base hogar con jefe informal se observa el mismo resultado que para Argentina, que el jefe tenga un trabajo formal aumenta la probabilidad de asistencia. Asimismo, el efecto negativo de la pertenencia a un hogar con hacinamiento también se verifica y lo mismo sucede con la incidencia positiva del clima educativo del hogar.

El análisis de la situación en Bahía Blanca se encuentra limitado por la escasa cantidad de observaciones. Un avance en el estudio de los determinantes del acceso a la educación media para esta población podría ser abordado a partir del relevamiento propio que forma parte del proyecto de Unidad Ejecutora del Instituto de Investigaciones Económicas y Sociales del Sur: "Inclusión social sostenible: Innovaciones y políticas públicas en perspectiva regional”3 (en proceso). 
María M. Ibáñez, María M. Formichella y Lucas E. Costabel

Cuadro 4. Determinantes de la probabilidad de asistir al nivel medio de educación. Barrios Bahía Blanca. Abril 2016

\begin{tabular}{|c|c|}
\hline Variables explicativas & Coeficientes \\
\hline \multirow[t]{2}{*}{ Edad } & $-0.2534^{\star \star \star}$ \\
\hline & (0.1528) \\
\hline \multirow[t]{2}{*}{ Sexo } & -0.8774 \\
\hline & $(0.0745)$ \\
\hline \multirow[t]{2}{*}{ Clima educativo } & $0.4554^{*}$ \\
\hline & $(0.1567)$ \\
\hline \multirow[t]{2}{*}{ Hogar monoparental } & -0.0706 \\
\hline & $(0.8284)$ \\
\hline \multirow[t]{2}{*}{ Adolescente activo } & $-5.2681^{\star}$ \\
\hline & $(0.9210)$ \\
\hline \multirow[t]{2}{*}{ Hacinamiento } & -1.3332 \\
\hline & (0.8674) \\
\hline \multirow[t]{2}{*}{ Cobertura en salud } & 1.0004 \\
\hline & $(0.6672)$ \\
\hline \multirow[t]{2}{*}{ Tarea doméstica } & -0.5658 \\
\hline & $(0.6568)$ \\
\hline \multirow[t]{2}{*}{ Hogar jefe trabajador inestable } & -0.4585 \\
\hline & (0.7229) \\
\hline \multirow[t]{2}{*}{ Adolescente cónyuge } & $-2.7458^{\star}$ \\
\hline & (1.063) \\
\hline \multirow[t]{2}{*}{ Constante } & 4.6719 \\
\hline & (3.0453) \\
\hline \multicolumn{2}{|c|}{$\begin{array}{l}\text { Notas: } * ; * * ; \\
\text { mente. Desvíos estándar entre paréntesis. }\end{array}$} \\
\hline \multicolumn{2}{|c|}{$\begin{array}{l}\text { Fuente: elaboración propia con base en EPH (INDEC, 2016), segundo trimestre } 2016 \\
\text { y con base en estimaciones realizadas en STATA 13.1. }\end{array}$} \\
\hline
\end{tabular}


Además, los hogares con mayor bagaje educativo aumentan las probabilidades de que sus miembros adolescentes asistan a la escuela y, en este caso, la variable hacinamiento no ha resultado ser estadísticamente significativa (aunque puede deberse a la baja variabilidad de la misma en la muestra).

Respecto a las variables referidas a la condición laboral del jefe de hogar, se incorpora sólo "jefe con trabajo inestable" debido a que no fue posible incluir las variables de jefe formal, informal y desocupado dada la cantidad de observaciones y variabilidad de estas variables en la muestra. ${ }^{4}$ En este caso, la condición de inestabilidad del jefe de hogar parece no ser relevante en la explicación de la probabilidad de asistencia escolar de los adolescentes de los barrios en cuestión.

De los resultados expuestos se desprende la existencia de cierta homogeneidad en la significatividad de las variables explicativas incorporadas. Para Argentina y el aglomerado Bahía Blanca-Cerri se verifica que la probabilidad de que un adolescente asista a un centro educativo se encuentra negativamente afectada por el avance de la edad, el hecho de ser hombre, pertenecer a un hogar con hacinamiento o monoparental, realizar tareas domésticas o estar activo laboralmente. Por el contrario, las posibilidades aumentan en caso de contar con cobertura en salud, pertenecer a hogares con jefes insertos en el mercado laboral formal o con mayor clima educativo. En el caso de los barrios enmarcados en el proyecto PDTS, se mantiene las significatividad y el sentido de las variables edad, clima educativo y adolescente activo.

\section{CONSIDERACIONES FINALES}

A lo largo de este trabajo se analizó la exclusión en su dimensión educativa, tomando en cuenta el primer paso de la misma, relacionada con las restricciones de acceso. Se consideraron los individuos en edad de asistir al nivel educativo medio (de 12 a 18 años) en tres poblaciones diferentes, con el fin de detectar si se evidencian elementos en común en los determinantes del proceso excluyente.

Se llegó a dos conclusiones principales: por un lado, las variables que resultaron ser relevantes en relación con la posibilidad de escolarización de los adolescentes coinciden con estudios previos en la materia y, por otro, ciertas variables mantienen su significatividad estadística en los tres casos de estudio considerados. 
A partir de los resultados, se observa que la no escolarización se acrecienta con la edad: los adolescentes logran ingresar al sistema educativo, pero no lo pueden sostener. Se detectan factores expulsores relacionados a la necesidad de tomar responsabilidades adultas en edades tempranas, tales como ser jefe de hogar, estar a cargo de las tareas domésticas o ser activo laboralmente. El tiempo es limitado y todas estas cuestiones compiten con el que puede ser dedicado a estudiar. Asimismo, de los resultados surge que existen elementos que "empujan" a los adolescentes a enfrentarse con este tipo de responsabilidades, entre ellos, habitar un hogar con algún tipo de carencia material, con un jefe que no puede asegurar una corriente continua y segura de ingresos o con un solo adulto a cargo. También, se observa que los individuos de género masculino son más vulnerables ante este tipo de situaciones.

Por otra parte, se destaca el rol de la educación de los padres y del clima educativo del hogar. A mayor nivel educativo de los adultos, mayor es la probabilidad de que los adolescentes asistan a la escuela. La correlación entre estas variables podría estar reflejando el efecto de la importancia que se le otorga a la educación de los menores en un hogar, bajo la premisa que adultos más educados valoran más la educación, así como que adultos más educados poseen más y mejores herramientas para acompañar los procesos de escolarización.

Respecto a la población que habita barrios vulnerables, se resalta el rol de la edad, de ser económicamente activo y de habitar un hogar con bajo clima educativo. Estas son las variables que resultaron ser significativas desde el punto de vista estadístico y coinciden con las que resultaron serlo en las otras dos poblaciones. Puede que otros de los elementos antes mencionados no hayan sobresalido en este contexto por falta de variabilidad.

En suma, en este trabajo se realizó un aporte al análisis de la exclusión educativa en su grado original (acceso) y en contextos diferentes. Queda abierta la puerta para avanzar sobre el estudio de otros grados de exclusión educativa, como en la búsqueda de elementos comunes que puedan evidenciarse en escenarios diversos. 


\section{BIBLIOGRAFÍA}

Alderete, M. V., Formichella, M. M. y Krüger, N. (2017), "Acceso y uso de TIC en barrios vulnerables de Bahía Blanca: su efecto sobre los resultados educativos", Anales de la LII Reunión Anual de la Asociación Argentina de Economía Política. Recuperado de <www.aaep.org.ar>

Atkinson, A. B. y Hills, J. (1998), "Exclusion, employment and opportunity", Research Paper, núm. CASE004, London School of Economics, London, Centre for Analysis of Social Exclusion. Recuperado de <http://repositorio.minedu.gob.pe/bitstream/handle/123456789/3533/Exclusion, $\% 20$ Employment\%20and\%20Opportunity.pdf?sequence=1>

Banco Interamericano de Desarrollo (вID) (2007), Ideas para el Desarrollo en las Américas (IDEA), "¿Los de afuera? La exclusión social en América Latina", vol. 14, Washington, Banco Interamericano de Desarrollo, septiembrediciembre.

Berghman, J. (1995), "Social exclusion in Europe: policy context and analytical framework", en G. Room (ed.), Beyond the threshold: the measurement and analysis of social exclusion, Bristol, Policy Press.

Bertranou, E. (2001), Determinantes del avance en los niveles de educación en Argentina: análisis empirico basado en un modelo probabilístico secuencial (Tesis de Maestría en Economía), Argentina, Universidad Nacional de La Plata.

Blanco, G. (2006), "La equidad y la inclusión social: uno de los desafíos de la educación y la escuela hoy", Revista Iberoamericana sobre Calidad, Eficacia y Cambio en Educación, vol. 4, núm. 3, Madrid, Red Iberoamericana de Investigación Sobre Cambio y Eficacia Escolar, julio-septiembre.

Brugué, Q., Gomà, R. y Subirats, J. (2002), "De la pobreza a la exclusión social. Nuevos retos para las políticas públicas", Revista Internacional de Sociología, núm. 33, Madrid, Consejo Superior de Investigaciones Científicas, septiembre-diciembre.

Burchardt, T. (1998), Submission to glasgow regeneration alliance social, inclusion inquiry, Documento de trabajo, Reino Unido, Universidad de Glasgow.

Burchardt, T., Le Grand, J. y Piachaud, D. (1999), "Social exclusion in Britain 1991-1995", Social Policy and Administration, núm. 33, New Jersey, Wiley- Blackwell, septiembre.

Castells, M. (2001), La conexión perversa: la economia criminal global. La era de la información, vol. 3, Buenos Aires, Fin de Milenio. 
Cerrutti, M. y Binstock, G. (2004), Camino a la exclusion: determinantes del abandono escolar en el nivel medio en la Argentina, I Congresso da Associação Latino Americana de Populaçáo, Brasil. Recuperado de <https:// studylib.es/doc/7286562/camino-a-la-exclusi\%C3\%B3n--determinantesdel-abandono-escolar>

Comisión de las Comunidades Europeas (1992), Hacia una Europa de la solidaridad. Intensificación de la lucha contra la exclusión social y la promoción de la integración, Bruselas, сом.

(2000), Construir una Europa que fomente la integración, Consejo Europeo de Lisboa, conclusiones de la presidencia, Lisboa. Recuperado de <http://www.europarl.europa.eu/summits/lis1_es.htm>

De la Puente, J. L. B. (2009), "Hacia una educación inclusiva para todos", Revista Complutense de Educación, vol 20, núm. 1, Madrid, Universidad Complutense de Madrid, septiembre-diciembre.

De Vos, S. (2001), "Family structure and school attendance among children 13-16 in Argentina and Panama", Journal of Comparative Family Studies, vol. 32, núm. 1, Toronto, University of Toronto Press.

Estivill, J. (2003), Panorama de la lucha contra la exclusión social: conceptos y estrategias, International Labour Organization, Suiza, Oficina Internacional del Trabajo. Recuperado de <http://www.derechoshumanos.unlp.edu. ar/assets/files/documentos/panorama-de-la-lucha-contra-la-exclusionsocial.pdf>

Fabre, M. M. (2000), "Consideraciones en torno al concepto de exclusión social”, Acciones e investigaciones sociales, núm. 11, España, Facultad de Ciencias Sociales y del Trabajo de la Universidad de Zaragoza, abril.

Formichella, M. M. (2010), Educación y desarrollo: análisis desde la perspectiva de la equidad educativa interna y del mercado laboral (Tesis de Doctoral), Argentina, Universidad Nacional del Sur.

(2011), "Exclusión y trabajo informal en la Argentina", Realidad Económica, núm. 264, Buenos Aires, Instituto Argentino para el Desarrollo Económico, agosto.

Formichella, M. M. y Krüger, N. (2019), "Condiciones socioeducativas heterogéneas en barrios periféricos de Bahía Blanca”, Revista de Ciencias Sociales, vol. 14, núm. 1, Costa Rica, Universidad de Costa Rica.

Formichella, M. M., Krüger, N. y Reyes, M. (2017), Condiciones socioeducativas heterogéneas en barrios periféricos de Bahía Blanca, Actas del VI Congreso Nacional e Internacional de Estudios Comparados en Educación. Disponible en <http://www.saece.com.ar/papers6.php> 
Gasparini, L. C. (2001), "On the measurement of unfairness: an application to high school attendance in Argentina”, Social Choice and Welfare, núm. 19, Alemania, Springer, diciembre.

Golovanevsky, L. (2003), Pobreza, vulnerabilidad y exclusión. Sus aportes diferenciales para la comprensión de la situación social de Jujuy (1991-2001), $6^{0}$ Congreso Nacional de Estudios del Trabajo. Recuperado de $<$ https://www. aset.org.ar/congresos/6/archivosPDF/grupoTematico06/006.pdf >

Groisman, F. (2011), "Argentina: los hogares y los cambios en el mercado laboral (2004-2009)", Revista CEPAL, núm. 104, México, Comisión Económica para América Latina y el Caribe, agosto.

(2012), "Determinantes de la escolarización y participación económica de los adolescentes en Argentina (2004-2009)", Frontera Norte, vol. 24, núm. 48, México, Colegio de la Frontera Norte, julio-diciembre.

Groisman, F. y Calero, A. V. (2010), Educación y participación económica de los jóvenes en Argentina. Un análisis de sus determinantes (2004-2009), XLV Reunión Anual de la Asociación Argentina de Economía Política. Recuperado de <https://aaep.org.ar/anales/works/works2010/groisman_cale ro.pdf>

Gujarati, D. y Porter, D. (2009), Econometría, México, McGraw-Hill.

Herrero, V. (2005), Determinantes de situaciones de riesgo educativo en la población en edad escolar en Argentina, VIII Jornadas Argentinas de Estudios de Población de la Asociación de Estudios de la Población de Argentina. Recuperado de <http://www.redaepa.org.ar/jornadas/viii/AEPA/B14/ Herrero.pdf>

Hopenhayn, M. (2008), "Inclusión y exclusión social en la juventud latinoamericana", Pensamiento Iberoamericano, núm. 3, Madrid, Fundación Carolina y Agencia Española de Cooperación Internacional. Recuperado de <http://red.pucp.edu.pe/wp-content/uploads/biblioteca/110616.pdf>

Ibáñez Martín, M. M. (2015), Segmentación e inequidad educativa en Argentina: su relación con la movilidad social (Tesis de Maestría), Argentina, Universidad Nacional del Sur. Recuperado de <http://repositoriodigital.uns.edu. ar/bitstream/123456789/2374/1/Tesis\%20de\%20Iba\%C3\%B1ez\%20 Martin.pdf>

(2018), Exclusión social: los desafios de su conceptualización y medición. Una propuesta desde un enfoque axiomático. Aplicación para Argentina (Tesis Doctoral), Argentina, Universidad Nacional del Sur. Recuperado de <http://repositoriodigital.uns.edu.ar/handle/123456789/ 4475> 
Ibáñez Martín, M. M., Formichella, M. M. y London, S. (2017), Exclusión social, desigualdad y desarrollo económico, IV Foro Bienal Iberoamericano de Estudios del Desarrollo, Quilmes, Argentina.

indec (2003), La nueva Encuesta Permanente de Hogares de Argentina, Argentina, INDEC. Recuperado de <https:/www.indec.gob.ar/ftp/cuadros/ sociedad/metodologia_eph_continua.pdf>

(2010), Resultados del Censo 2010. Recuperado de <https://www.indec.gob.ar/indec/web/Nivel4-Tema-2-41-135>

(2014), Sistema de estadisticas sociodemográficas. Recuperado de <https://www.indec.gov.ar/nuevaweb/cuadros/7/sesd_glosario.pdf>

(2016), Base de microdatos de la Encuesta Permanenete de Hogares 2016. Recuperado de <https:/www.indec.gob.ar/indec/web/Institucional-Indec-BasesDeDatos>

Jiménez Zambrano, G. (2005), "Modified maximum likelihood estimation of tobit models with fixed effects: theory and an application to earnings equations", Investigaciones Económicas, vol. 29, núm. 3, España, Fundación SEPI.

Kessler, G. (2011), “Exclusión social y desigualdad ¿nociones útiles para pensar la estructura social argentina?”, Lavboratorio, núm. 24, Buenos Aires, Instituto de Investigaciones Gino Germani, septiembre.

(2014), Controversias sobre la desigualdad: Argentina, 2003-2013, Argentina, Fondo de Cultura Económica.

Kristensen, H. (1995), "Social exclusion and spatial stress. The connections", en G. Room (ed.), Beyond the threshold: the measurement and analysis of social exclusion, Bristol, Policy Press.

Krüger, N. (2013), Equidad educativa interna y externa en Argentina: un análisis para las últimas décadas (Tesis Doctoral), Argentina, Universidad Nacional del Sur.

Látas, Á. P. (2002), "Acerca del origen y sentido de la educación inclusiva”, Revista de educación, núm. 327, Mar del Plata, Facultad de Humanidades, Universidad Nacional de Mar del Plata, marzo.

Ley 26.206 (2006), Ley de Educación Nacional. Boletín Oficial, 28 de diciembre de 2006. Recuperado de: http://servicios.infoleg.gob.ar/infolegInternet/verNorma.do?id=123542

Liao, T. F. (1994), Interpreting probability models: logit, probit and other generalized linear models (Sage University Paper series on Quantitative Applications in the Social Sciences, series no. 07-101), Thousand Oaks, Sage.

Lipton, M., Yaqub, S. y Darbellay, E. (1998), Successes in anti-poverty, Geneva, International Labour Office. 
Marshall, T. H. (1964), Class, citizenship and social development, New York, Greenwood Press.

Minujin, A. (1999), “¿La gran exclusión? Vulnerabilidad y exclusión en América Latina”, en D. Filmus (comp.), Los noventa: politica, sociedad y cultura en América Latina y Argentina de fin de siglo, Buenos Aires, FLACso/Eudeba.

Miranda, A. (2010), "Educación secundaria, desigualdad y género en Argentina", Revista Mexicana de Investigación Educativa -RMIE, núm. 45, México, Consejo Mexicano de Investigación Educativa, abril-junio.

Miranda, A., Otero, A. y Corica, A. (2007), "Cambio y situación social de los jóvenes en Argentina”, Papeles de población, vol. 13, núm. 52, México, Universidad Autónoma del Estado de México, Centro de Investigación y Estudios Avanzados de la Población, abril-junio.

Morresi, S., Ibáñez Martín, M. M. y Arnaudo, M. F. (2017), Inclusión educativa: ¿las nuevas universidades nacionales colaboran en el proceso inclusivo?, SAECE. Recuperado de <http://www.saece.com.ar/docs/congreso6/ trab079.pdf>

Narodowski, M. (2008), "La inclusión educativa. Reflexiones y propuestas entre las teorías, las demandas y los slogans", Revista Iberoamericana sobre Calidad, Eficacia y Cambio en Educación, vol. 6, núm. 2, Madrid, Red Iberoamericana de Investigación sobre Cambio y Eficacia Escolar.

Paz, J. A. y Cid, J. C. (2012), "Determinantes de la asistencia escolar de los jóvenes en la Argentina", Revista electrónica de investigación educativa, vol. 14, núm. 1, Baja California, Instituto de Investigación y Desarrollo Educativo de la Universidad Autónoma de Baja California, octubre.

Pindyck, S. y Rubinfeld, L. (1998), Economentric models and economic forecasts, United States of America, McGraw-Hill, Inc.

Rama, G. W. (1983), "La educación latinoamericana: exclusión o participación”, Revista de la CEPAL, núm. 21, México, Comisión Económica para América Latina y el Caribe.

Rivero, J. (1999), Educación y exclusión en América Latina: reformas en tiempos de globalización, Lima, Tarea.

Sánchez, P. A. (2012), "Luchando contra la exclusión: buenas prácticas y éxito escolar", Innovación Educativa, núm. 21, Santiago de Compostela, Universidad de Santiago de Compostela.

Sarrionandia, G. E. (2006), Educación para la inclusión o educación sin exclusiones, Madrid, Narcea, S. A. de Ediciones.

Sen, A. (1999), Desarrollo y libertad, España, Editorial Planeta. 
Serio, M. (2017), "Desigualdad de oportunidades educativas en Argentina", Archivos Analiticos de Políticas Educativas, vol. 25, núm. 121, Arizona, Arizona State University.

Silver, H. (1994), "Culture, politics and national discourses of the new urban poverty", en E. Mingione (ed.), Urban poverty and the underclass, Oxford, Blackwell.

Siteal (2015), Base de indicadores estadísticos. Descripción. Recuperado de $<$ http://www.siteal.iipe.unesco.org/indicadores>

Sosa, W. y Marchionni, M. (1999), Household structure, gender, and the economic determinants of school attendance in Argentina, Documentos del Banco Mundial, núm. 37583. Recuperado de <http://documents.worldbank.org/ curated/en/999431468205791084/Household-structure-gender-and-theeconomic-determinants-of-school-attendance-in-Argentina>

Terigi, F., Perazza, R. y Vaillant, D. (2010), Segmentación urbana y educación en América Latina. El reto de la inclusión escolar, Madrid, FIECC.

Tezanos, J. F. (1999), El contexto sociopolitico de los procesos de exclusión social. Tendencias en desigualdad y exclusión social, Madrid, Editorial Sistema.

UNESCO (2006), Guidelines for inclusion: ensuring access to education for all, París, unEsCo.

Velasco, M. S. (1996), "La regresión logística. Una aplicación a la demanda de estudios universitarios”, Estadística Española, vol. 38, núm. 141, Madrid, Instituto Nacional de Estadísticas España.

Vitello, S. J. y Mithaug, D. E. (1998), Inclusive schooling: national and international perspectives, New Jersey, Lawrence Erlbaum Associates.

Williams, R. (2006), "Generalized ordered logit/partial proportional odds models for ordinal dependent variables", The Stata Journal, vol. 6, núm. 1, Notre Dame, University of Notre Dame, febrero.

Wooldridge, J. M. (2010), Introducción a la Econometría. Un enfoque moderno, México, South-Western Cengage Learning. 